\title{
Powder Injection Moulding of Multi-Material Devices
}

\author{
V. Piotter, E. Honza, A. Klein \\ T. Mueller, K. Plewa
}

Karlsruhe Institute of Technology (KIT)

Institute for Applied Materials (IAM-WPT) 


\section{Contents}

- $\quad$ Introduction / Objectives

- $\quad$ Two-component-PIM

Material combinations

\section{Assembly-PIM}

- $\quad$ Inmould-Labelling-PIM

- Hybrid Process Combinations

- $\quad$ Summary and Outlook 


\section{Driving Forces for Multi-Material PIM}

\begin{tabular}{|c|c|}
\hline Economical Objectives & Technological Objectives \\
\hline create high value-add products & $\begin{array}{c}\text { create innovative products } \\
\text { with properties profile }\end{array}$ \\
\hline $\begin{array}{c}\text { reduction of assembly expenditure } \\
\text { low costs in large and medium } \\
\text { series production }\end{array}$ & \\
\hline $\begin{array}{c}\text { equipment based on established } \\
\text { 2C-machinery }\end{array}$ & \\
\hline
\end{tabular}




\section{Multimaterial Devices => Multifunctional Products}

with complimentary or contradictionary properties, e.g.

\author{
conductive \\ hard \\ magnetic \\ hydrophilic \\ dense
}

$\leftrightarrow$

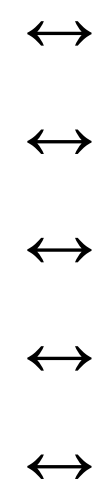

etc. insulating

tough non-magnetic hydrophobic porous 


\section{Driving Forces for Multi-Material PIM}

\begin{tabular}{|c|c|}
\hline Economical Objectives & Technological Objectives \\
\hline create high value-add products & $\begin{array}{c}\text { create innovative products } \\
\text { with properties profile }\end{array}$ \\
\hline $\begin{array}{c}\text { reduction of assembly expenditure } \\
\text { strong and tight } \\
\text { material connections }\end{array}$ \\
\hline $\begin{array}{c}\text { low costs in large and medium } \\
\text { series production }\end{array}$ & $\begin{array}{c}\text { several sub-variants } \\
\text { of basic process }\end{array}$ \\
\hline $\begin{array}{c}\text { equipment based on established } \\
\text { 2C-machinery }\end{array}$ & \\
\hline
\end{tabular}


further

component(s)

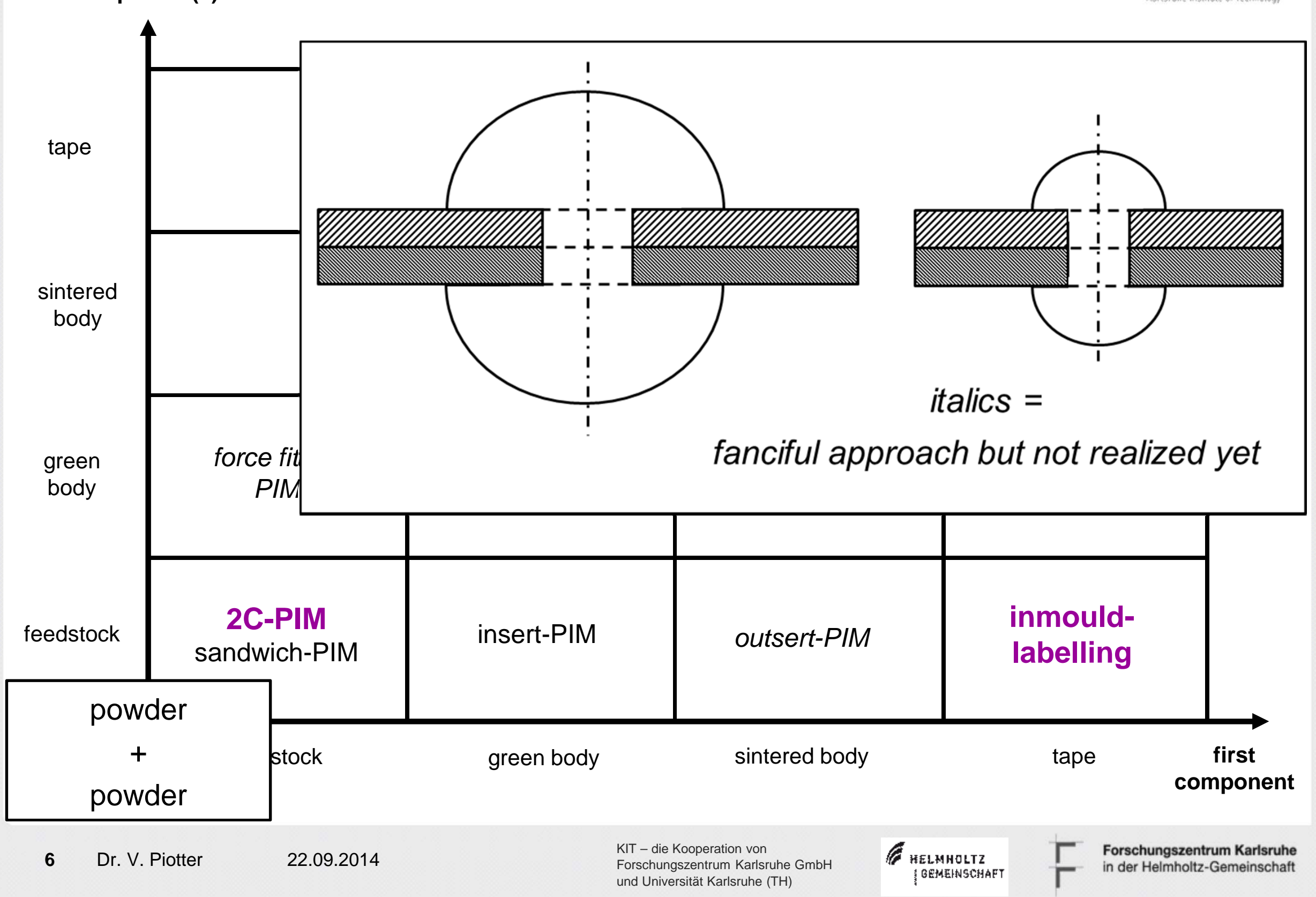

$\mathbb{V}<I T$

\section{Multi-Material PIM}




\section{2-Component PIM (Overmoulding)}

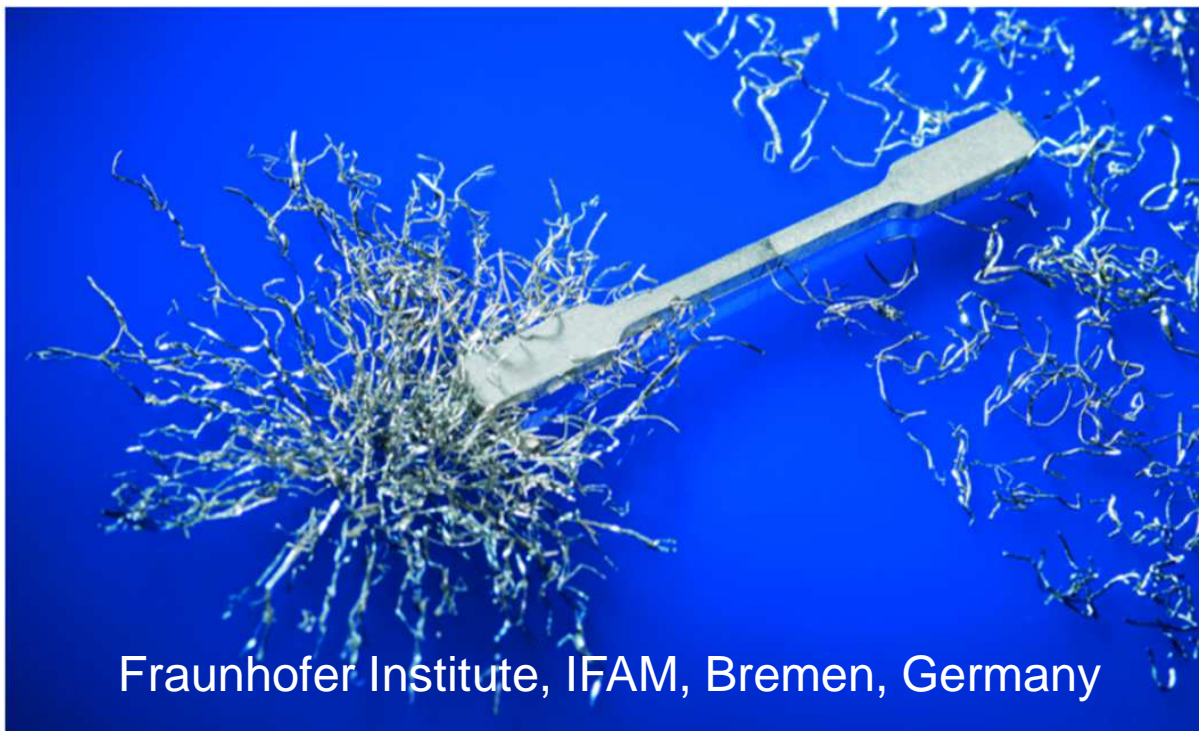

Combination of a magnetic steel (17-4PH)

with a non-magnetic steel (316L)

Hard metal WCxCo with different Co-contents (16\% and 6\%) ARBURG
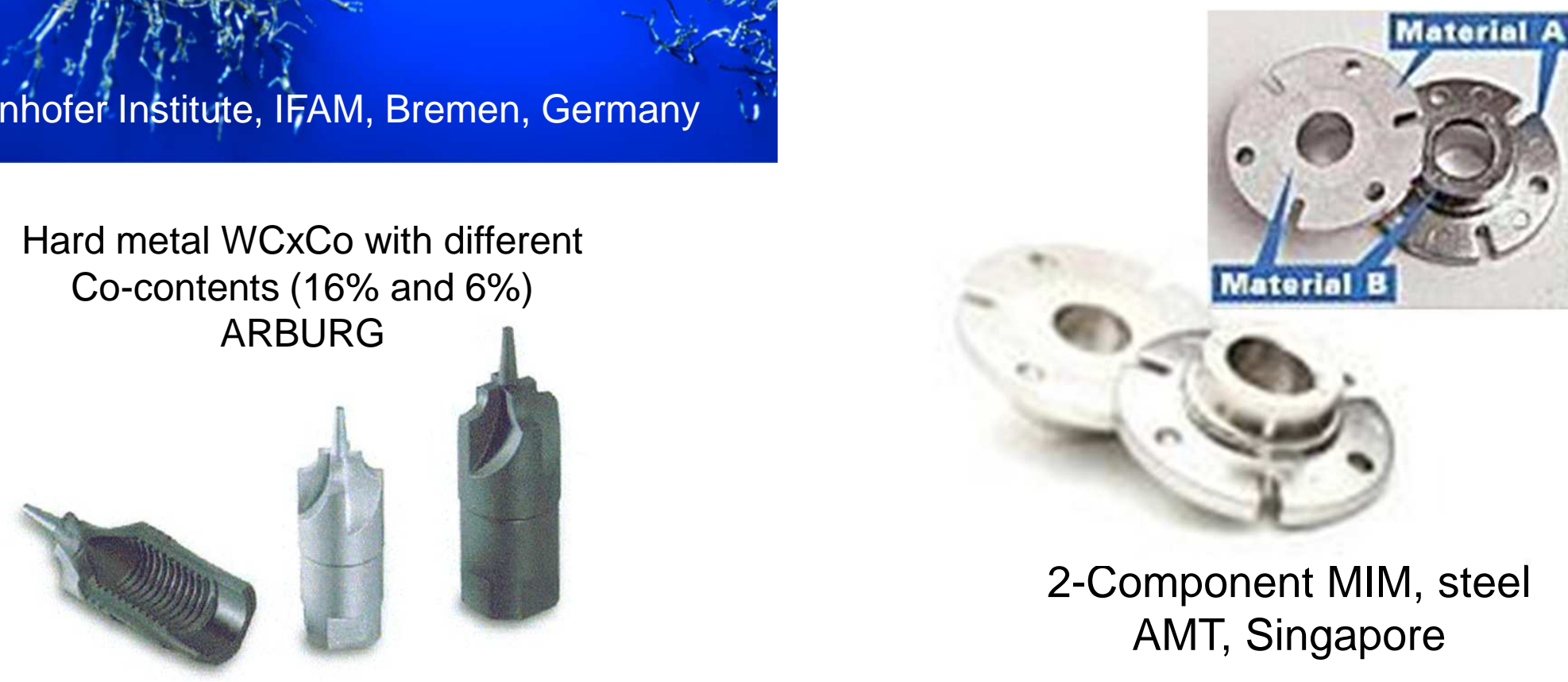


\section{2-Component Tungsten PIM (2C-WPIM)}
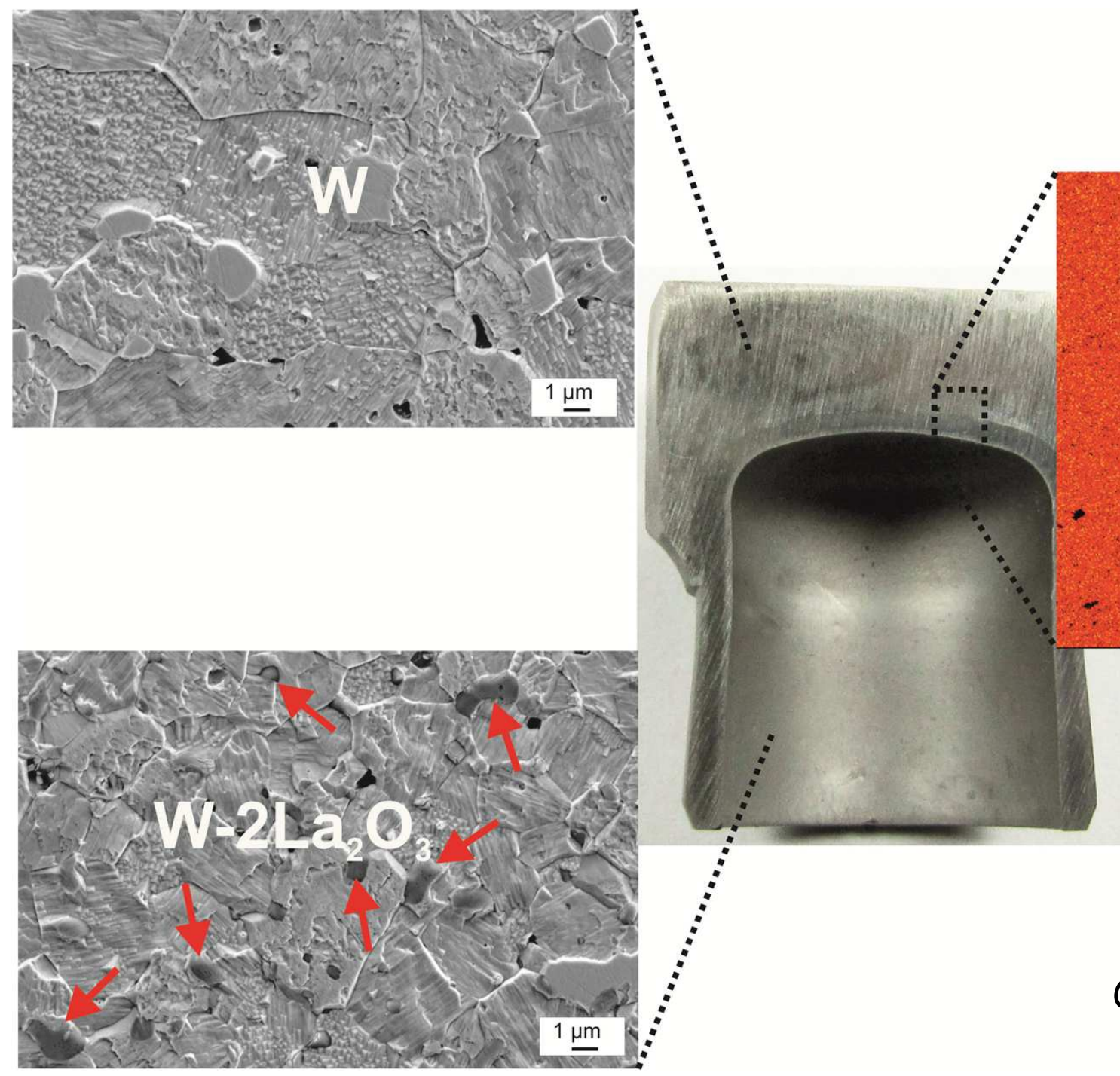

\section{AES Map \\ black: $\mathrm{La}_{2} \mathrm{O}_{3}$ red: $W$ $\mathrm{O}_{3}$}




\section{Combine different Material Classes}

Fixed connection of metal (steel 430L) and ceramic $\left(\mathrm{ZrO}_{2}\right)$

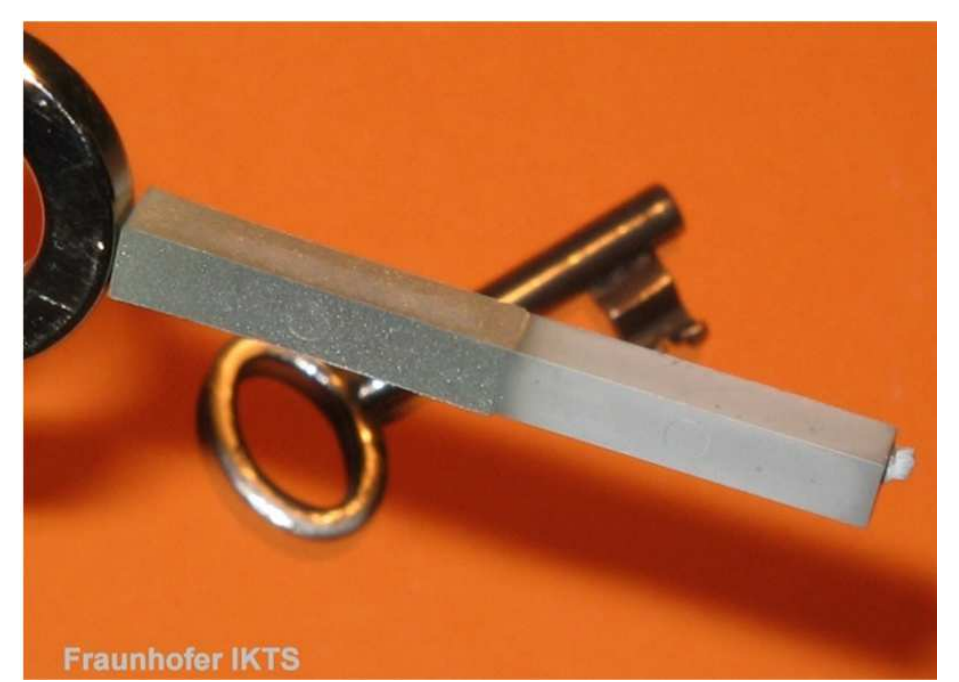

BSEM images of the interface of ceramic $\left(\mathrm{ZrO}_{2}\right)$ and metal (steel 17-4PH) samples Courtesy of Fraunhofer Institute IKTS, Dresden, Germany

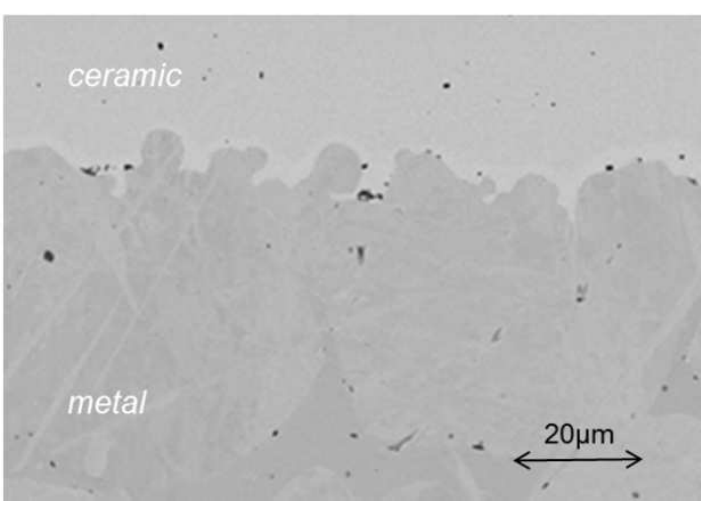

microscopic interlocking structure supported by a partial material bond

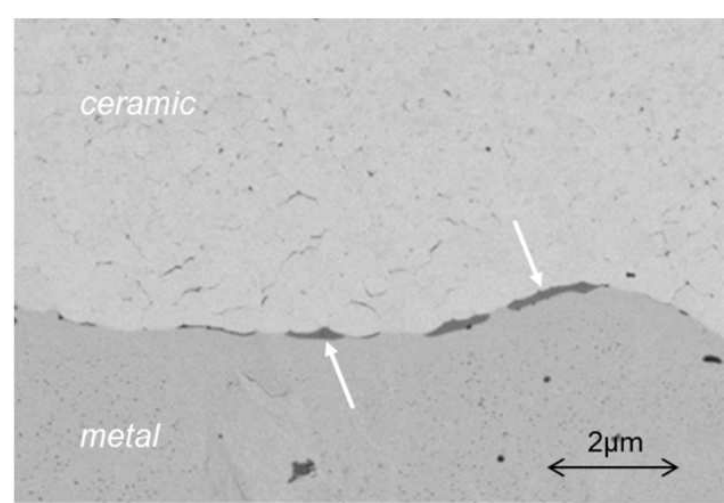

intermediate phase can be detected (white arrows) 


\section{Assembly Powder Injection Moulding}

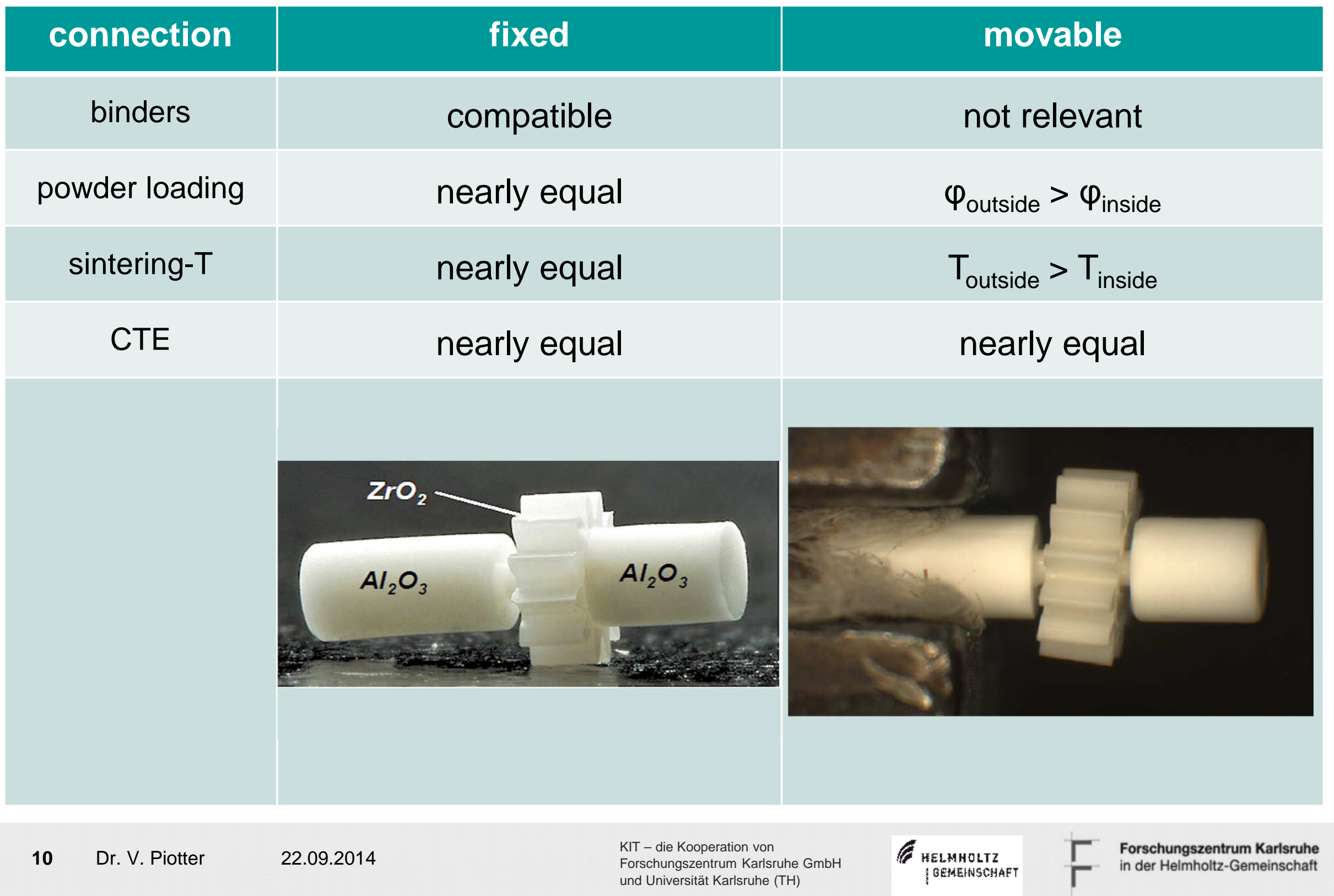


Fixed connections of different ceramics

Realisation of movable connections
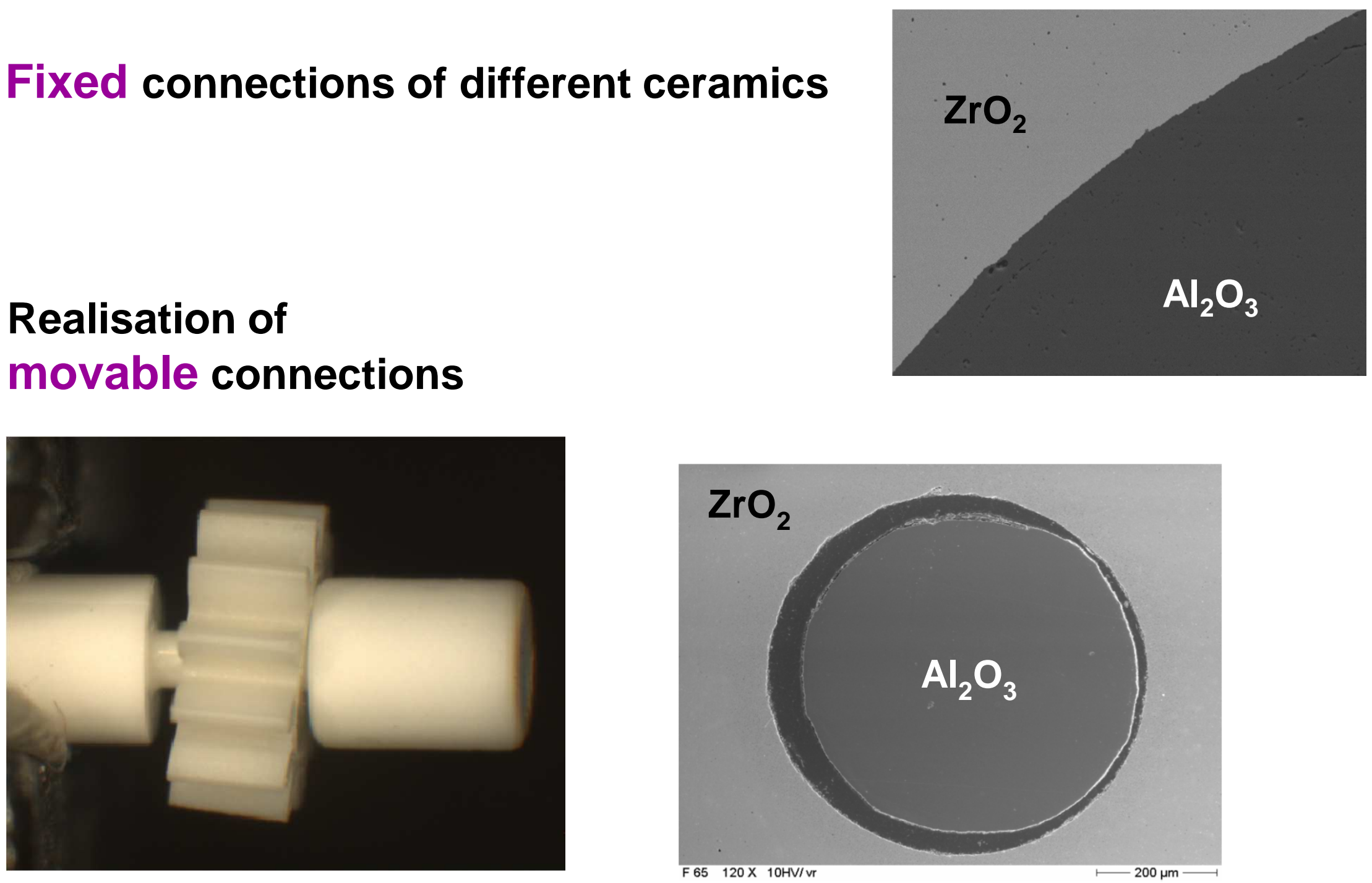
PIM green bodies + non-PIM green bodies

\section{Powder Inmould-labelling (IML-PIM)}
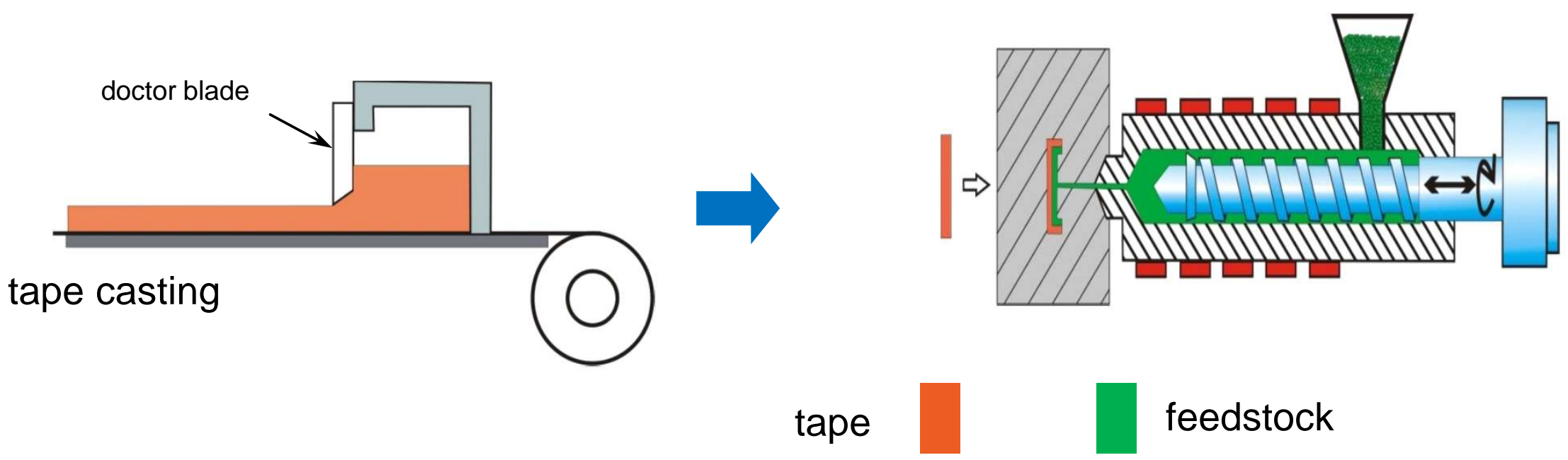

\section{EU Project No. FP7-NMP4-2007-214122}




\section{Micro Powder Inmould-labelling}

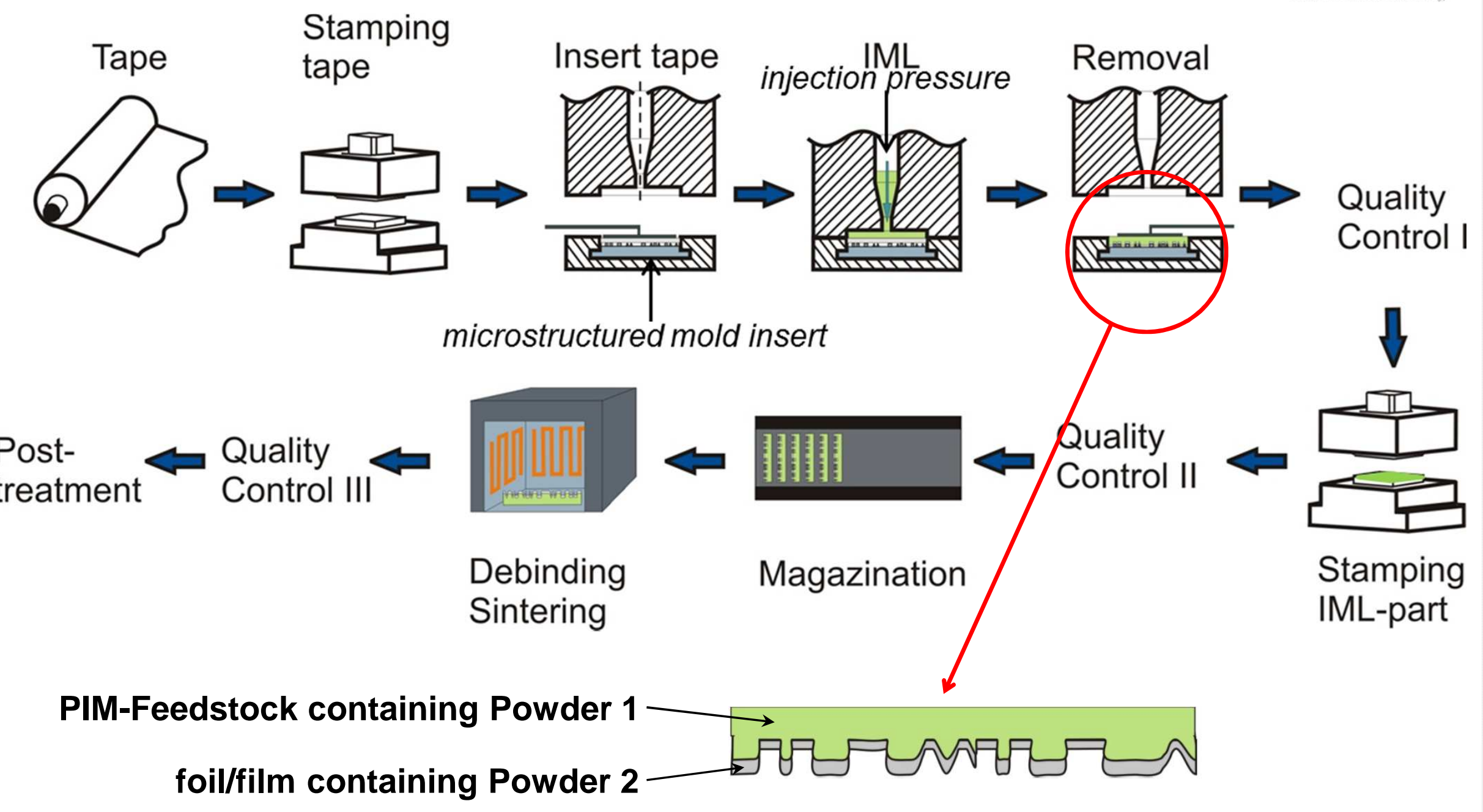

Powder 2: functional or nano-particles applied on the structured surface 


\section{Investigation of samples}

foil

green body

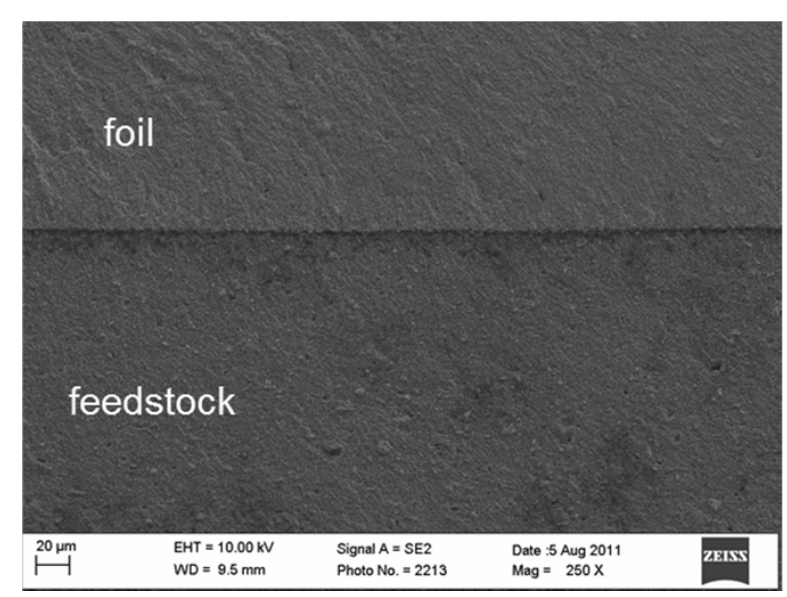

ca. $53 \mathrm{Vol} \% \mathrm{ZrO}_{2}$

$70 \mathrm{~nm}$

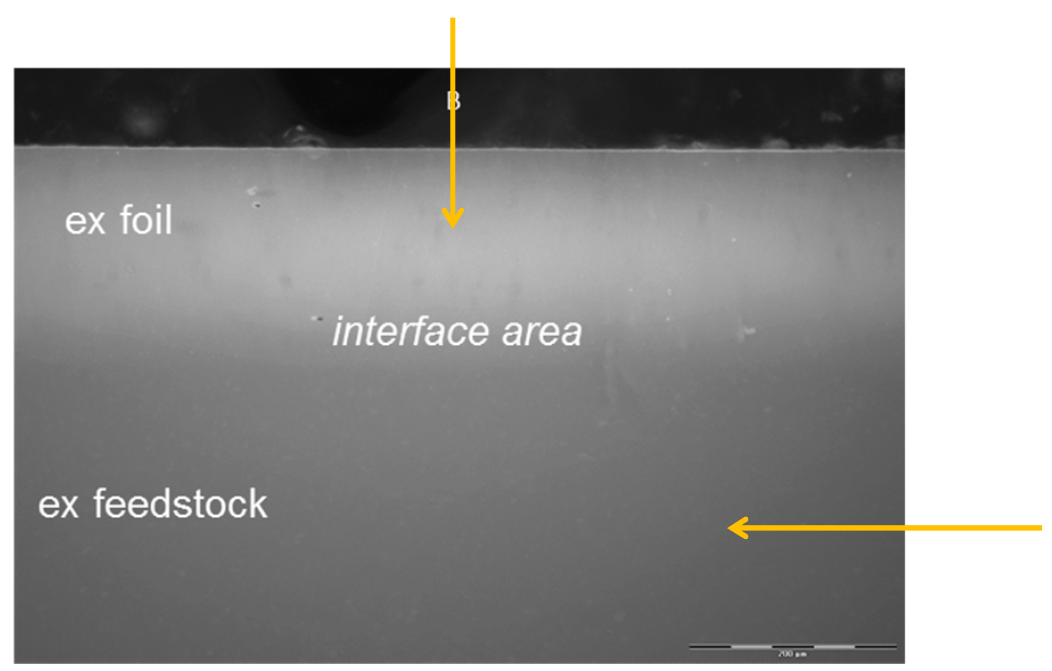

ca. $50 \mathrm{Vol} \% \mathrm{ZrO}_{2}$ $440 \mathrm{~nm}$

ca. $50 \mathrm{Vol} \% \mathrm{ZrO}_{2}$ $40 \mathrm{~nm}$

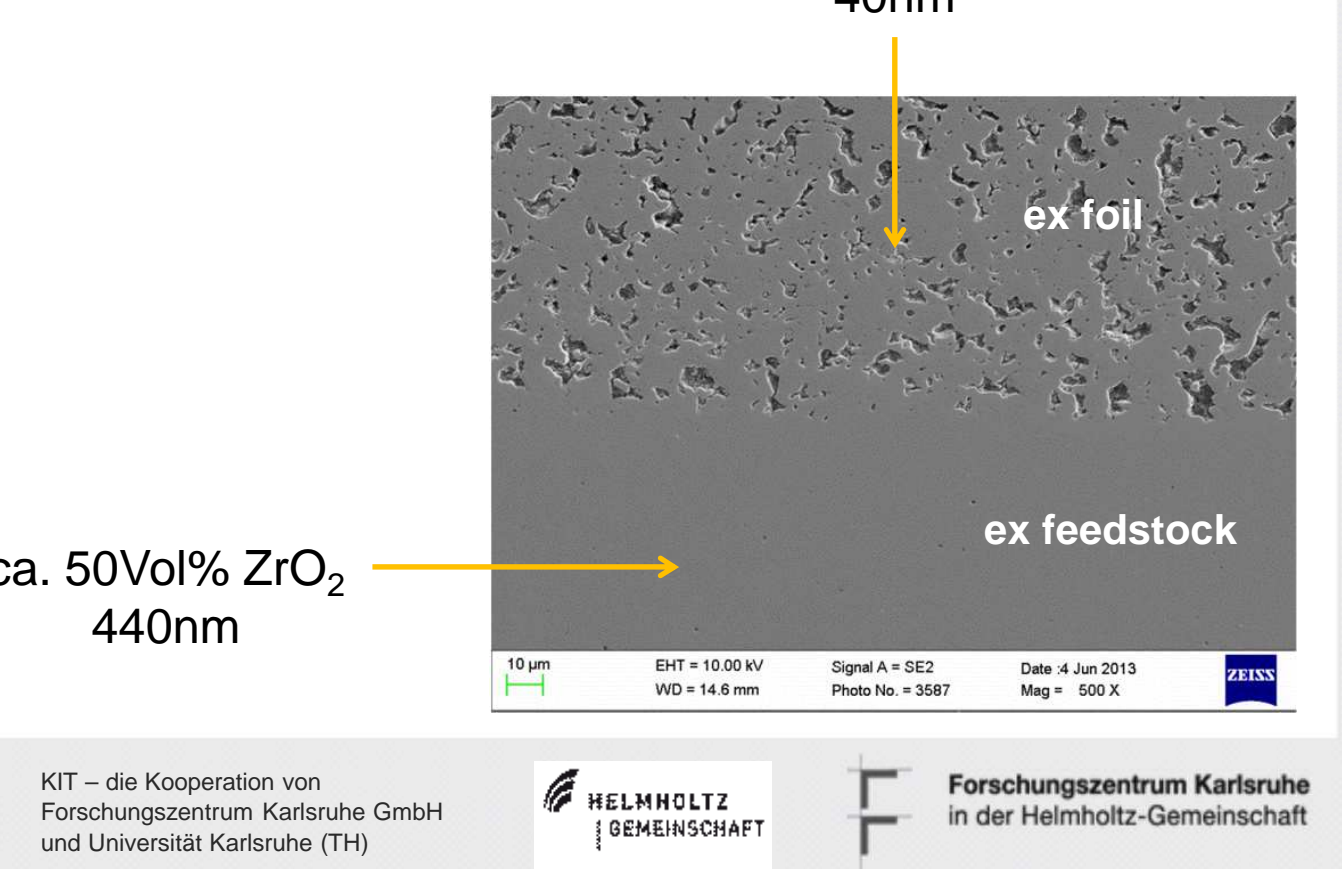




\section{Hybrid Process Combinations}

PIM green bodies + non-moulded materials

PIM + Additive Manufacturing

1. Micro PIM

2. 3D inkjet printing

3. Debinding

4. Sintering

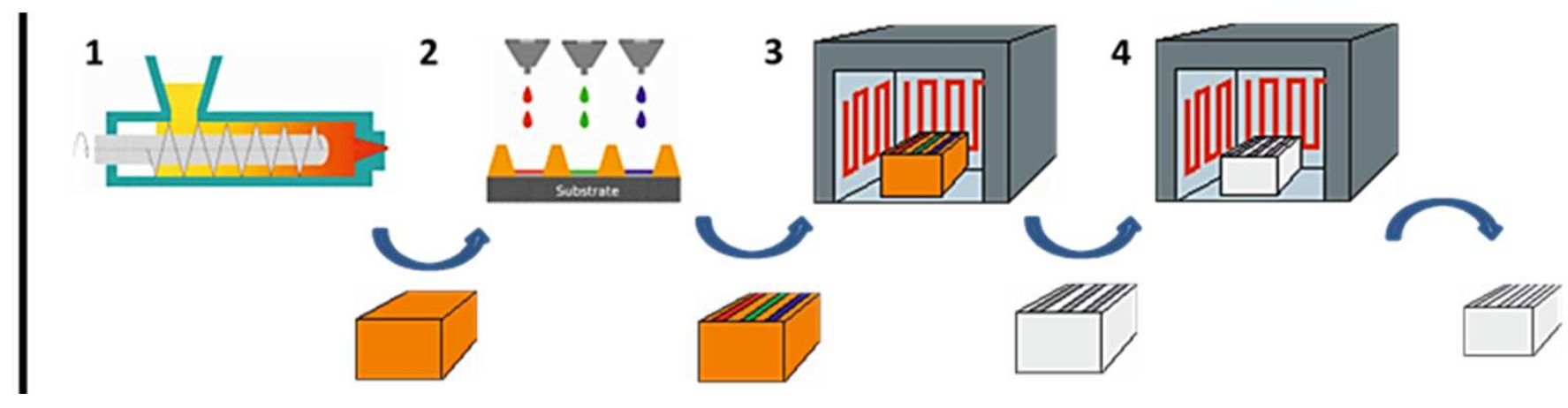

Combining the advantages of

PIM (high throughput) + AM (customized complex parts) 


\section{Summary and Outlook}

- $\quad$ Benefits of Multi-Material PIM

economical and technological

- $\quad$ Possibilities of Process Combinations

ideas and realized approaches

- $\quad$ Examples for Material Combinations

2C-WPIM, metal/ceramic joints

- $\quad$ Current focus on Two-component PIM

inmould-labelling PIM 


\section{Acknowledgment}

- $\quad$ Fraunhofer Institutes IKTS and IFAM

- $\quad$ Federal Ministry for Education and Research BMBF

- $\quad$ European Commission

- $\quad$ Deutsche Forschungsgemeinschaft DFG (SFB 499)

- Companies Arburg, microParts, Wittmann Battenfeld, SPT Roth, Sigma Engineering, Junghans, OBE etc.

- $\quad$ All colleagues at KIT

\section{Thank you!}

